\title{
How to manage children with anogenital warts
}

\author{
Margaret Kingston, ${ }^{1}$ Denise Smurthwaite, ${ }^{2}$ Sarah Dixon, ${ }^{3}$ Catherine White ${ }^{4}$
}

${ }^{1}$ The Hathersage Integrated Contraception, Sexual Health \& HIV Service, Manchester Royal Infirmary, Central Manchester Foundation Trust, Manchester, UK

${ }^{2}$ St Mary's Sexual Assault Referral Centre, Central Manchester Foundation Trust, Manchester, UK

${ }^{3}$ Paediatrics, Royal Manchester Children's Hospital, Central Manchester Foundation Trust, Manchester, UK

${ }^{4}$ St Mary's Sexual Assault Referral Centre, Central Manchester Foundation Trust, Manchester, UK

\section{Correspondence to}

Dr Margaret Kingston, Manchester Royal Infirmary, Central Manchester Foundation Trust, Manchester Centre for Sexual Health, The Hathersage Centre, Manchester M13 OFH, UK;

margaret.kingston@cmft.nhs.uk

Received 13 August 2015 Revised 25 February 2016 Accepted 5 March 2016 Published Online First 6 April 2016

\section{Sinked}

- http://dx.doi.org/10.1136/ sextrans-2015-052473

CrossMark

To cite: Kingston $\mathrm{M}$ Smurthwaite D, Dixon S, et al. Sex Transm Infect 2017;93:267-269.
The case

A 9-year-old girl is referred by her general practitioner to genitourinary medicine for the treatment of anogenital warts. The referral letter states that she is an otherwise fit and well girl taking no medicines.

-What issues should be considered here?

- Are any tests required, and if so which?

-What about treatment?

\section{INTRODUCTION}

Children found to have anogenital warts (AGW) are usually brought in the first instance to their general practitioners (GPs), who may assess and manage the children themselves or refer them onto a specialist from one of several disciplines including paediatricians, dermatologists, paediatric surgeons or genitourinary medicine (GUM) physicians. In addition, if child sexual abuse (CSA) is considered advice may be sought from a sexual assault referral centre (SARC), and if so referral to children's social care and police would be advised.

The authors have all been contacted for advice regarding the management of these children and we have noted:

- Variable degrees of competence in the diagnosis of AGW.

- Unfamiliarity with paediatric genital examination.

- Unfamiliarity with the need to test for coexisting sexually transmitted infections (STIs) in children in whom CSA is suspected.

- Lack of up-to-date knowledge regarding appropriate tests including those for other STIs.

- Variable levels of concern about possible CSA and in some cases lack of awareness that this diagnosis requires consideration and what action is then required.

To streamline this process, provide a framework for safeguarding evaluation and to ensure that all children are appropriately assessed, we developed a clinical algorithm for clinicians in any field supported by an appendix on sampling for STI screening for those less familiar with this.

\section{METHODS}

1. We reviewed the relevant specialty guidance on the management of STIs in children from:

A. The British Association for Sexual Health and HIV (BASHH) ${ }^{1}$

B. The physical signs of CSA publication from the Royal College of Paediatric and Child Health $^{2}$

2. We canvassed the opinion of other doctors in our specialties of GUM, paediatrics and forensic medicine, including the lead authors of the
BASHH guideline, by face-to-face meetings, email and telephone conversations.

3. The initial draft of the clinical algorithm evolved empirically with input from national guideline authors and local GP and colleagues from allied fields referred to in the introduction.

\section{RESULTS}

The current guidelines recognise a positive association between AGW and CSA and recommend that this must be considered in any child presenting with these. ${ }^{12}$ Sexual abuse is more likely to be confirmed in older prepubertal children in whom vertical transmission can be excluded as a possible cause.

Our final clinical algorithm is presented as a simple flowchart in figure 1 , and is now in use in our region.

\section{DISCUSSION}

While developing this algorithm we

encountered the following issues:

- Marked differences in the professional opinions regarding the significance of AGW in children and how children with genital warts should be assessed.

- Inconsistent onward referral pathway from primary care.

- A lack of robust evidence regarding age range compatible with vertical transmission.

- A lack of robust evidence regarding the possibility of inoculation of the child's immature genital skin by appropriate digital contact with others; nappy changing in very young children for example, or auto-inoculation of human papillomavirus (HPV) from non-genital wart virus infection.

- Deciding whether or not to test for and type the HPV virus. We decided against this for a number of reasons including:

- Lack of evidence that this is a clinically useful test when making a diagnosis and assessing children with genital warts.

- The tropism of HPV subtypes for different types of epithelium is not absolute, and this is not always applicable to the immature genital epithelium of children.

- HPV detection is dependent on when, and from which exact site, the swab is taken.

- Considerations for concurrent STI testing:

- The newer less invasive tests such as the nucleic acid amplification tests for Chlamydia trachomatis and Neisseria gonorrhoea and dried blood spot or oral fluid testing for antibodies to bloodborne viruses and syphilis have made this much easier and acceptable to children and to non-GUM doctors. 


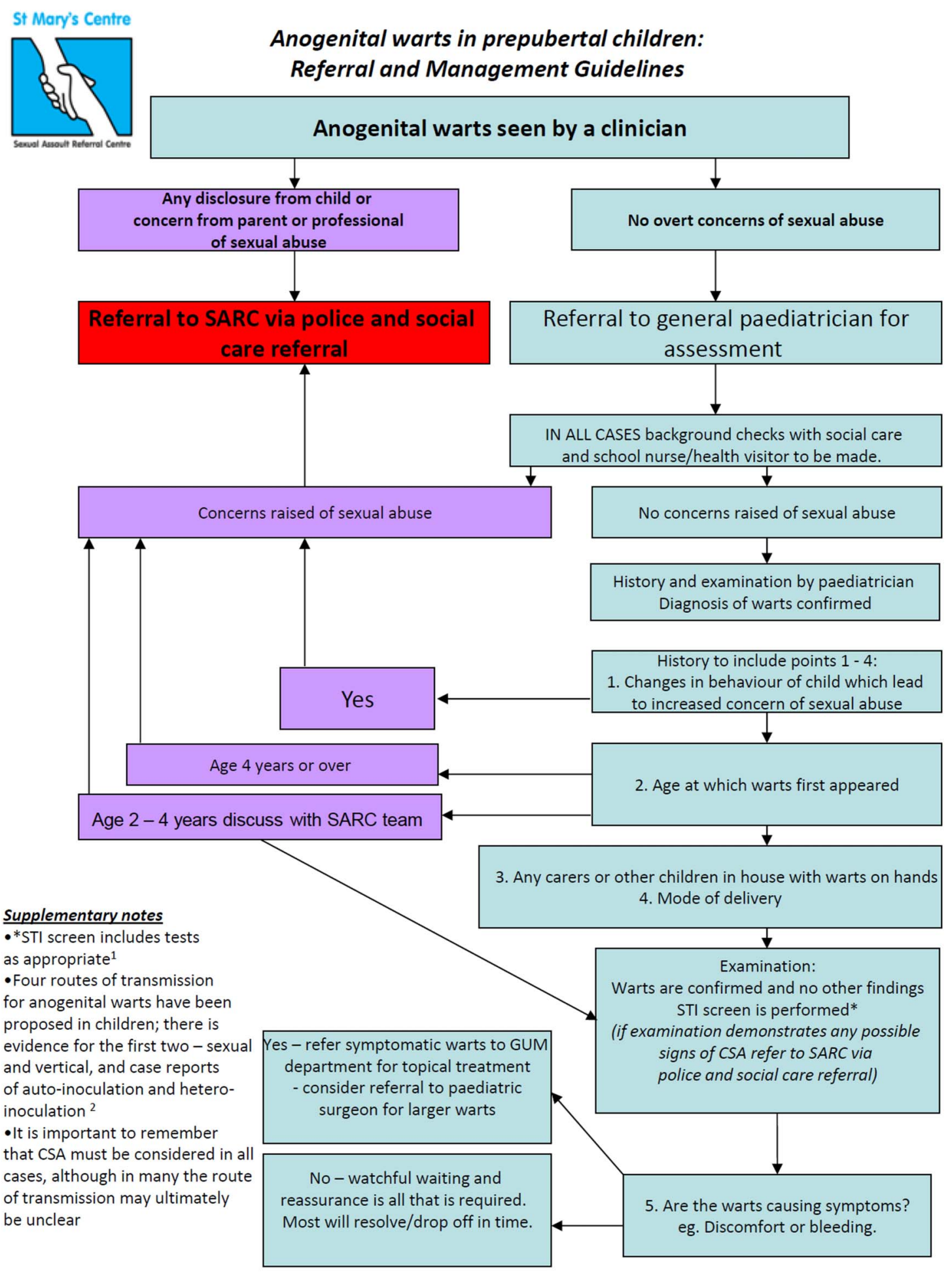

References: 1. UK National Guideline on the Management of Sexually Transmitted Infections and Related Conditions in Children and Young People - $\mathbf{2 0 1 0}$ http://www. bashh.org/guidelines/2. The Physical Signs of Child Sexual Abuse, 2015, RCPCH

Figure 1 Clinical algorithm for managing children with genital warts. CSA, child sexual abuse; GUM, genitourinary medicine; SARC, sexual assault referral centre; STI, sexually transmitted infection.

- The enhanced sensitivity and reliability of these tests mean that they are helpful when considering the likelihood of abuse-if concurrent STIs are found, this indicates CSA and heightens the likelihood that the AGW are due to this.

\section{CONCLUSIONS}

What are the benefits of using this algorithm?

- It endorses the need for specialist referral of all children from general practice.

- It supports effective interdisciplinary working. 
- Its supports non-GUM specialists in effective STI screening thus preventing the need for referral to GUM for the sole purpose of STI screening.

- This algorithm provides a secure framework for clinical and safeguarding, decision making that takes into account the uncertainties involved in assessing children with AGW and the variable experience and expertise in doctors to whom they may present or be referred to.

- Notwithstanding the limitations of the evidence base in this clinical area, we have found this to be a pragmatic approach which allows clinicians to assess children with an infection which may have been acquired through sexual contact.

\section{Back to the case}

What issues do you need to consider here?

Following the algorithm, the child would have been referred by her GP to paediatrics for assessment-this includes:

1. Background checks with social care and/or school nurses. Universal health service includes allocation of a health visitor for every child up to the fifth birthday and a school nurse thereafter until age 16 years. Social care will advise if there are any pre-existing concerns about the child, siblings and/or parents relevant to establish an index of suspicion of possible CSA.

2. History and examination covering the points in the algorithm in figure 1. Visual examination by an experienced GUM physician is adequate to confidently diagnose AGW in most cases, and joint examination with paediatric and GUM, where possible, is ideal. In a minority of cases where the appearance is atypical, the examination under magnification (usually using a colposcope) or failing that a biopsy may be required. ${ }^{3}$

3. As she is aged over 4 years, a referral to SARC via social care (or the police depending on the details of the case) for investigation of possible sexual abuse is indicated.

\section{Do you need to take any tests, and if so which are you going to} do?

1. Paediatric assessment includes an STI screen with samples from sites according to any disclosure of sexual abuse or as it thought appropriate.

2. Serologic testing for syphilis and bloodborne viruses should be considered, and dried blood spot or oral fluid testing may be preferable to venous blood sampling.

3. Testing for HPV is not helpful.

Finally, what about treatment?

Treatment is required if the warts are causing problematic symptoms. Depending on the type and location of the warts and the preference of the child and their carers, a referral to GUM for medical or to paediatric surgery for operative treatment can be offered. Ablative treatment, such as cryotherapy (with topical anaesthetic agents), is an option ${ }^{1}$ but may be limited by acceptability to the child and family as this may be distressing for the child and require repeat attendances. Topical treatment of AGW in children with imiquimod is unlicensed in children and not recommended in the summary of product characteristics. However, when studied for the treatment of molluscum contagiosum in children, while demonstrating no efficacy for this condition, neither were there any serious adverse events and local side effects were similar to those in adults. ${ }^{4}$ Case reports and case series have reported successful treatment of AGW with imiquimod $^{5-8}$ which is consistent with our experience of treating these children, with full explanation and informed consent from the child (where appropriate and possible) and the person with parental responsibility for them.

Suppose the case had been that of an 18-month-old boy-would you do anything differently?

The GP would still refer to a paediatrician for history, examination, STI screening and assessment including background checks as detailed in the algorithm. Valid consent from the person with parental responsibility for the child is required to undertake STI screening. Informed consent requires that the clinician makes clear that the reason for the STI screen is to investigate the possibility that the genital warts have resulted from CSA. If there is no cause for concern, you would then advise observation or treatment depending on symptoms.

\section{What are the next steps for our group?}

1. This algorithm has been incorporated into our local clinical practice, and we are very happy to share it with other relevant clinicians and guideline authors.

2. A local course has been established to improve the confidence of doctors who need to undertake genital examination in children (St Mary's SARC annual study day on anogenital findings in children; http://www.stmaryscentre.org).

3. We have developed local guidance on sampling for STIs in children.

4. We are prospectively evaluating the application of this pathway in our services.

\section{Handling editor Jackie A Cassell}

Contributors All authors have given relevant advice and themselves managed children with anogenital warts and at risk of child sexual abuse, and as a consequence of their clinical experiences worked collaboratively to develop this clinical management algorithm. All have contributed to writing this article.

Competing interests None declared.

Provenance and peer review Commissioned; externally peer reviewed.

\section{REFERENCES}

1 United Kingdom National Guideline on the Management of Sexually Transmitted Infections and Related Conditions in Children and Young People-2010. Karen Rogstad, Amanda Thomas, Olwen Williams, Greta Forster, Pat Munday, Angela Robinson, Guy Rooney, Jackie Sherrard, Melinda Tenant-Flowers, Dawn Wilkinson, Neil Lazaro. http://www.bashh.org/BASHH/Guidelines/Guidelines/BASHH/Guidelines/ Guidelines.aspx (accessed 23 Feb 2016).

2 The Physical Signs of Child Sexual Abuse. Royal College of Paediatrics and Child Health. 2015.

3 UK National Guidelines on the Management of Anogenital Warts. 2015. http://www. bashh.org/documents/UK\%20national\%20guideline\%20on\%20Warts\%202015\% 20FINAL.pdf (last accessed 23 Feb 2016).

4 SPC Aldara 5\% cream. https://www.medicines.org.uk/emc/medicine/8 (accessed 23 Feb 2016).

5 Dinleyici M, Saracoglu N, Eren M, et al. Giant Condyloma Acuminate Due to Human Papillomavirus Type 16 in an Infant Successfully Treated with Topical Imiquimod Therapy. Dermatol Rep 2015;7:6134.

6 Sikanić Dugić N, Ljubojević Hadžavdić S, Pustišek N, et al. Treatment of anogenital warts in an 18-month-old girl with 5\% imiquimod cream. Acta Dermatovenerol Croat 2014;22:40-3

7 Grussendorf-Conen El, Jacobs S. Efficacy of imiquimod $5 \%$ cream in the treatment of recalcitrant warts in children. Pediatr Dermatol 2002;19:263-6.

8 Moresi JM, Herbert CR, Cohen BA. Treatment of anogenital warts in children with topical $0.05 \%$ podofilox gel and $5 \%$ imiquimod cream. Pediatr Dermatol 2001; 18:448-50. 